\title{
Comment on 'Energy spectrum of a Dirac particle with position-dependent mass under the influence of the Aharonov-Casher effect ${ }^{*}$
}

\author{
V. B. Mendrot ${ }^{\dagger}$ and A. S. de Castro ${ }^{\ddagger}$ \\ Departamento de Física, \\ Universidade Estadual Paulista "Júlio de Mesquita Filho", \\ Guaratinguetá, SP, Brazil
}

\begin{abstract}
It is shown that the paper 'Energy spectrum of a Dirac particle with positiondependent mass under the influence of the Aharonov-Casher effect', by Oliveira, Borges and Sousa [Braz. J. Phys. 49, 801 (2019)], is based on a series of ingredients clearly incorrect.
\end{abstract}

Keywords: Dirac particle . position-dependent mass . chirality . $(2+1)$-dimensional world

\section{Introduction}

In the paper 'Energy spectrum of a Dirac particle with position-dependent mass under the influence of the Aharonov-Casher effect', recently published in the Brazilian Journal of Physics [1], Oliveira, Borges and Sousa described the planar motion of a structured neutral fermion with position-dependent mass embedded in a $(2+1)$-dimensional world instead of a $(3+1)$-dimensional Minkowski space-time.

Possible difficulties regarding the ordering of position and momentum operators in the Schrödinger equation with a position-dependent mass has nothing to do with a positiondependent mass in the Dirac equation. In the context of the Dirac equation, positiondependent mass serves as a means for expressing the mass of the fermion $m$ plus a positiondependent scalar interaction, and its nonrelativistic limit is the Schrödinger equation for a particle of mass $m$ under the influence of an effective position-dependent potential.

It is true that the anomalous magnetic moment of a structured neutral fermion can be modeled by the nonminimal electromagnetic tensor interaction in $3+1$ dimensions. Indeed, in $3+1$ dimensions the Dirac spinor has four components and the most general

\footnotetext{
*To appear in Brazilian Journal of Physics (DOI: 10.1007/s13538-020-00820-4)

${ }^{\dagger}$ E-mail: vitor.mendrot@unesp.br

${ }^{\ddagger}$ E-mail: antonio.castro@unesp.br
} 
interaction potential matrix without derivative couplings can be written in terms of sixteen linearly independent matrices arranged in terms of well defined Lorentz structures: scalar (1), pseudoscalar (1), vector (4), pseudovector (4) and tensor (6). Nevertheless, the little $(2+1)$-dimensional space-time is a very diverse world: the Dirac spinor has two components and there are only four two-by-two linearly independent matrices which can be grouped in terms well defined Lorentz structures: scalar (1) and vector (3).

To adjust knowledge gained through experience in $3+1$ dimensions to fit knowledge in $2+1$ dimensions, and vice-versa, might be a source of danger. For example, the fourcomponent spinor in $3+1$ dimensions takes into account the two energy states and the two spin states of the fermion for given momentum. How to accommodate the two energy states and the two spin states of the fermion using a two-component spinor? In 1978, de Vega pointed out that the spin of a fermion in a $2+1$-dimensional space-time is not an independent degree of freedom and that the state can be completely specified by its momentum and sign of the energy [2].

The authors of Ref. [1], as a starting point for their calculations in $2+1$ dimensions, built "left-handed" and "right-handed" projection operators with a "chirality" operator which does not anticommutes with all the $\gamma$-matrices appearing in the Dirac equation. The authors of Ref. [1], though, making reference to a book which treats the Dirac equation only in $3+1$ dimensions, took those anticommutation relations for granted.

Trying to obtain a quadratic form of the Dirac equation, increasing by a factor of two the number of solutions sought, the authors of Ref. [1] failed to consider the derivative property of the momentum operator.

Furthermore, without any justification, the authors of Ref. [1] selected one of the two possible nonequivalent representations of the $\gamma$-matrices.

The purpose of this comment is to present and urge reasons in opposition to a number of lapses in judgment by the authors of Ref. [1]. Those slips concerning vital relations for the development of their work make their conclusions discredited.

\section{The Dirac equation in a $(2+1)$-dimensional world}

In order to clarify our criticisms, let us begin writing the Dirac equation in a $(2+1)$ dimensional space-time. The Dirac equation for a fermion of mass $m$ and momentum $p^{\mu}=\left(p^{0}, \mathbf{p}\right)$ is written (in natural units $\hbar=c=1$ ) as

$$
\left(\gamma^{\mu} p_{\mu}-m I-V\right) \Psi=0
$$

where $\mu=0,1,2, p_{\mu}=i \partial_{\mu}, V$ is the interaction potential matrix and $I$ is the unit matrix. The state of the system is specified by the spinor $\Psi$. The $\gamma$-matrices satisfy the algebra $\left\{\gamma^{\mu}, \gamma^{\nu}\right\}=2 g^{\mu \nu} I$, where $g^{\mu \nu}$ stands for the matrix element of the Minkowski metric tensor with $g^{00}=-g^{i i}=1$ and $g^{\mu \nu}=0$ if $\mu \neq \nu$. The $\gamma$-matrices can be represented by twoby-two matrices, and the unit matrix plus the three Pauli matrices $\sigma_{i}$ form the base of the vector space of all $2 \times 2$ matrices. In general each $\gamma^{\mu}$ possesses four matrix elements so that we should be able to form four, and only four, independent quantities with them. Usually, the basis elements $\Gamma$ are chosen in such a way that the bilinear $\Psi^{\dagger} \gamma^{0} \Gamma \Psi$ has a definite transformation property under Lorentz transformations. We find scalar and vector structures in $2+1$ dimensions (in the sense of proper Lorentz transformations) in such a way that the most general interaction potential matrix with direct (nonderivative) 
couplings can be written as $V=\gamma^{\mu} A_{\mu}+I S$. The four real quantities $A_{\mu}$ and $S$ are called vector and scalar potentials, respectively, due to the transformation properties under Lorentz transformations of the bilinear forms $\Psi^{\dagger} \gamma^{0} \gamma^{\mu} \Psi$ and $\Psi^{\dagger} \gamma^{0} I \Psi$. It is instructive to note the simple way that $A_{\mu}$ and $S$ make their appearance from the Dirac equation for a free fermion: $p_{\mu} \rightarrow p_{\mu}-A_{\mu}$ and $m \rightarrow m+S$. Up to unitary transformations, two specific representations can be constructed as $\gamma^{0}=\sigma_{3}$ and $\boldsymbol{\gamma}=\sigma_{3} \boldsymbol{\sigma}$, with $\boldsymbol{\sigma}=\left(\sigma_{1}, s \sigma_{2}\right)$ and $s= \pm 1$, so that the two-component Dirac equation can be compactly written as

$$
\left[I\left(p_{0}-A_{0}\right)-\boldsymbol{\sigma} \cdot(\mathbf{p}-\mathbf{A})-\sigma_{3}(m+S)\right] \Psi=0 .
$$

Using the identity $\sigma_{i} \sigma_{j}=\delta_{i j} I+i \sum_{k} \varepsilon_{i j k} \sigma_{k}$, where $\delta_{i j}$ is the Kronecker delta and $\varepsilon_{i j k}$ is the Levi-Civita symbol, we can write $\boldsymbol{\sigma} \cdot \mathbf{A}=i s \boldsymbol{\sigma} \cdot \sigma_{3} \mathbf{a}$, where $\mathbf{a}=\left(A^{2},-A^{1}\right)$, in such a way that we end up with [3]

$$
\left[I\left(p_{0}-A_{0}\right)-\boldsymbol{\sigma} \cdot\left(\mathbf{p}-i s \sigma_{3} \mathbf{a}\right)-\sigma_{3}(m+S)\right] \Psi=0 .
$$

The interaction potential matrix related to the space component of a vector potential $A^{\mu}$ has been replaced by that one with the same matrix structure of the Dirac oscillator [4] but with a more general potential function a. Those two different values of $s$ give rise to two nonequivalent representations of the $\gamma$-matrices (the solutions of the Dirac equation are $s$-dependent as can be seen, for example, in Ref. [4]).

\section{Criticisms}

In Ref. [1], with the $\gamma$-matrices expressed in terms of the Pauli matrices, the authors referred to

$$
\left[\gamma^{\mu} p_{\mu}-m(\mathbf{r})\right] \Psi(t, \mathbf{r})=0
$$

as the Dirac equation of a free spin-1/2 particle with position-dependent mass, but in plain terms it actually describes a fermion subject to a position-dependent scalar interaction. See Eq. (16) in Ref. [1] for a particular case of $m(\mathbf{r})$. There is no free particle described by Eq. (4), as might be expected.

For including the Aharonov-Casher effect, the authors of Ref. [1] considered a tensor potential which might be written as a space component of a vector potential.

Serving as an essential ingredient of their work, the authors of Ref. [1] defined "left-handed" and "right-handed" projection operators by $P_{L}=\left(I-\gamma^{5}\right) / 2$ and $P_{R}=$ $\left(I+\gamma^{5}\right) / 2$, where, they said, $\gamma^{5}=\sigma_{1}$. However, the cited property $P_{R} \gamma^{\mu}=\gamma^{\mu} P_{L}$ would be true only if $\left\{\gamma^{5}, \gamma^{\mu}\right\}=0$. This anticommutation relation, though, is never satisfied because there is no room for a fourth matrix anticommutating with all the $\gamma$-matrices appearing in the Dirac equation.

Considering the desideratum indispensable property $P_{R} \gamma^{\mu}=\gamma^{\mu} P_{L}$ (it does not accord with the truth, we insist), by defining $\Psi_{R}(t, \mathbf{r})=P_{R} \Psi(t, \mathbf{r})$ the quadratic Dirac equation should be written as

$$
\left[\gamma^{\mu} p_{\mu}-m(\mathbf{r})\right] \frac{1}{m(\mathbf{r})}\left[\gamma^{\mu} p_{\mu}+m(\mathbf{r})\right] \Psi_{R}(t, \mathbf{r})=0
$$

and not

$$
\left[\gamma^{\mu} p_{\mu}-m(\mathbf{r})\right]\left[\gamma^{\mu} p_{\mu}+m(\mathbf{r})\right] \Psi_{R}(t, \mathbf{r})=0
$$


Eq. (6) is the incorrect form found in Ref. [1] (see Eq. (5) in Ref. [1]). In this case, the authors of Ref. [1] transgressed for not considering that the momentum operator is a derivative operator acting on $m(\mathbf{r})$. It seems that they have forgotten what they said in the middle of Sec. 1 of their work: "...the mass $m(\mathbf{r})$ is an operator, it does not commute more with the moment operator...". As if that were not enough, the authors supported themselves in references $([55,56])$ that really do not address the problem of position-dependent mass.

The quadratic Dirac equation furnishes twice the number of the solutions sought but the authors of Ref. [1] did not say how to get rid of those spurious solutions.

The authors of Ref. [1] selected one of the two possible nonequivalent representations of the $\gamma$-matrices but they did not justify their choice.

\section{Final remarks}

With fulcrum on the precedent considerations, the embarrassing mistakes in Ref. [1] make it impossible to be confident about the conclusions manifested there.

\section{Acknowledgement}

Grant 2019/06734-2, São Paulo Research Foundation (FAPESP). Grant 09126/2019-3, Conselho Nacional de Desenvolvimento Científico e Tecnológico (CNPq), Brazil.

\section{References}

[1] R.R.S. Oliveira, V.F.S. Borges, M.F. Sousa, Energy spectrum of a Dirac particle with position-dependent mass under the influence of the Aharonov-Casher effect, Braz. J. Phys. 49, 801 (2019)

[2] H.J. de Vega, Fermions and vortex solutions in Abelian and non-Abelian gauge theories, Phys. Rev. D 18, 2932 (1978)

[3] A.S. de Castro, Equivalence between the Dirac oscillator and a spin-1/2 fermion embedded in a transverse homogeneous magnetic field: movement in a $(2+1)$ dimensional world, Rev. Bras. Ens. Fis. 42, e20190133 (2020)

[4] V.M. Villalba, Exact solution of the two-dimensional Dirac oscillator, Phys. Rev. A 49, 586 (1994) 